\title{
Totally positive extensions and weakly isotropic forms
}

\begin{abstract}
The aim of this article is to analyse a new field invariant, relevant to (formally) real fields, defined as the supremum of the dimensions of all anisotropic, weakly isotropic quadratic forms over the field. This invariant is compared with the classical $u$-invariant and with the Hasse number. Furthermore, in order to be able to obtain examples of fields where these invariants take certain prescribed values, totally positive field extensions are studied.
\end{abstract}

\section{Introduction}

The study of the $u$-invariant is a central topic in the theory of quadratic forms over fields. If $F$ is a nonreal field, then $u(F)$ is defined as the supremum of the dimensions of all anisotropic quadratic forms over $F$. Over a (formally) real field $F$ there exist always anisotropic quadratic forms of arbitrarily high dimension. In order to extend the above definition to obtain an invariant which is interesting for real fields too, one idea is to take the supremum of the dimensions of certain anisotropic quadratic forms subject to some extra condition which is trivial in the nonreal case. For example, one may consider only quadratic forms which are torsion, or totally indefinite. The two choices lead, respectively, to the definition of the $u$-invariant, as it was defined by Elman-Lam in [2], and the Hasse number. These two invariants have been extensively studied (cf. [7, Chap. 8]). However, there is at least one further important class of quadratic forms over real fields, the weakly isotropic ones. This class gives rise to a field invariant $\hat{u}$ defined as the supremum of the anisotropic forms in the class. For a general account on field invariants of this type, see [4]. In this article I want to initiate an examination of the invariant $\hat{u}$. This will be done in Section 5, after some preparation.

Central simple algebras and their Schur indices provide an important tool in the investigation of the (usual) $u$-invariant of a field. This is due to a relation between the dimension of the anisotropic part of a quadratic form and the index of its Clifford invariant. This relation was first used by Merkurjev in his construction of fields with prescribed even $u$-invariant. In order to be able to perform a similar construction for the new $\hat{u}$-invariant, I consider the pythagorean index of a central simple

K.J. Becher: Fachbereich Mathematik und Statistik, D203, Universität Konstanz, 78457 Konstanz, Germany. e-mail: becher@maths.ucd.ie

Classification (MSC 2000): 11E04, 11E81, 12D15 
algebra, defined as the Schur index of the algebra after extension of scalars to the pythagorean closure of its center. This invariant and its behaviour under certain field extensions is studied in Section 4. Before, some simple facts about totally positive field extensions need to be collected in Section 3.

\section{Terminology}

In the following I summarize what will be needed from the theory of quadratic forms and from real algebra. Details can be found in [6], [11], and [10].

Throughout this article, let $F$ be a field of characteristic different from 2. Let $F^{\times}$denote the multiplicative group of $F$ and $\sum F^{\times 2}$ the subgroup of non-zero sums of squares in $F$. The field $F$ is said to be real if $-1 \notin \sum F^{\times 2}$, otherwise $F$ is nonreal. If $F$ is nonreal, then $\sum F^{\times 2}=F^{\times}$.

An ordering of $F$ is a subset $P \subset F$ which is closed under addition and under multiplication and such that $P \cup-P=F$ and $P \cap-P=0$. Let $X(F)$ denote the set of all orderings of $F$. The Artin-Schreier Theorem says that $\cap X(F)=$ $\sum F^{\times 2} \cup\{0\}$, in particular $F$ is real if and only if it admits an ordering. The elements of $\sum F^{\times 2}$ are referred to as the totally positive elements of $F$.

By a 'form' or a 'quadratic form' I always mean a regular quadratic form. A quadratic form over $F$ may be given by a diagonalization $\left\langle a_{1}, \ldots, a_{n}\right\rangle$, with entries $a_{1}, \ldots, a_{n} \in F^{\times}$, which determines the form up to isometry. Let $W F$ denote the Witt ring of $F$, consisting of the Witt equivalence classes of quadratic forms over $F$, and $I F$ the fundamental ideal of $W F$, given by the forms of even dimension. For $n \geq 1$, let $I^{n} F$ denote its $n$th power $(I F)^{n}$.

Let $\varphi$ be a quadratic form over $F$. If $\varphi$ represents the zero element non-trivially over $F$, then $\varphi$ is said to be isotropic over $F$, otherwise anisotropic. If $\varphi$ represents all non-zero elements of $F$, then $\varphi$ is said to be universal. In particular, any isotropic form is universal.

For $m \in \mathbb{N}$, let $m \times \varphi$ denote the $m$-fold orthogonal sum $\varphi \perp \ldots \perp \varphi$. If $\varphi$ is isometric to $i \times\langle 1,-1\rangle$ for some $i \in \mathbb{N}$, then $\varphi$ is said to be hyperbolic. If there exists $m \geq 1$ such that $m \times \varphi$ is isotropic (resp. hyperbolic), then $\varphi$ is said to be weakly isotropic (resp.torsion). If $\varphi$ is not weakly isotropic, then we say that it is strongly anisotropic. Obviously, every non-trivial torsion form is weakly isotropic. If $F$ is a nonreal field, then actually every quadratic form over $F$ is torsion and, therefore, weakly isotropic.

A semiordering of $F$ is a subset $S \subset F$ such that $1 \in S, F^{2} S \subset S, S+S \subset S$, $S \cup-S=F$ and $S \cap-S=0$. Hence an ordering of $F$ is a semiordering $S$ of $F$ which further satisfies $S \cdot S \subset S$. The collection of all semiorderings of $F$ is denoted by $Y(F)$. Semiorderings on $F$ exist if and only if $F$ is a real field. Given a semiordering $S \in Y(F)$, the form $\varphi$ is indefinite at $S$ if $\varphi$ represents nonzero elements of $S$ and of $-S$. It is well-known that a quadratic form $\varphi$ over $F$ is weakly isotropic if and only if it is indefinite at every semiordering of $F$ (cf. [10, (2.9) Theorem]). A generally weaker condition is that $\varphi$ is indefinite at every ordering of $F$, in which case $\varphi$ is said to be totally indefinite. Hence weakly isotropic forms are totally indefinite. The converse is true for all quadratic forms over $F$ if and only if $F$ is a $S A P$-field. 
For any field extension $K / F$, let $\varphi_{K}$ denote the quadratic form $\varphi$ considered as a form over $K$.

If $\operatorname{dim}(\varphi) \geq 2$ and if $\varphi$ is not isometric to the hyperbolic plane $\mathbb{H}=\langle 1,-1\rangle$, then let $F(\varphi)$ denote the function field of the projective quadric over $F$ given by $\varphi$. If $\operatorname{dim}(\varphi) \leq 1$ or if $\varphi \cong \mathbb{H}$, then let $F(\varphi)=F$. Note that, if $\operatorname{dim}(\varphi) \geq 2$, then $\varphi_{F(\varphi)}$ is isotropic.

Let us define three field invariants:

$$
\begin{aligned}
& u(F)=\sup \{\operatorname{dim}(\varphi) \mid \varphi \text { anisotropic torsion form over } F\}, \\
& \hat{u}(F)=\sup \{\operatorname{dim}(\varphi) \mid \varphi \text { anisotropic, weakly isotropic form over } F\}, \\
& \tilde{u}(F)=\sup \{\operatorname{dim}(\varphi) \mid \varphi \text { anisotropic, totally indefinite form over } F\} .
\end{aligned}
$$

The suprema are taken in the (well-ordered) set $\mathbb{N} \cup\{\infty\}$. It is immediately clear that $u(F) \leq \hat{u}(F) \leq \widetilde{u}(F)$ for any field $F$ and that all three numbers are equal if $F$ is nonreal.

The first of these invariants is the $u$-invariant in its nowadays commonly accepted definition, due to Elman and Lam (cf. [2]). The last invariant $\tilde{u}$ was introduced by Prestel in [9] and called the Hasse number. A large part of what is known about these invariants can be found in [7, Chap. 8] and in [5]. The invariant $\hat{u}$ has not been studied so far. This shall be initiated in Section 5, where the mutual relationships between the three invariants will be discussed.

\section{Totally positive field extensions}

A field extension $K / F$ will be said to be totally positive if every semiordering of $F$ extends to a semiordering of $K$. This is trivially the case if $F$ is nonreal.

Lemma 3.1. For a field extension $K / F$, the following are equivalent:

(1) $K / F$ is totally positive.

(2) For any quadratic form $\varphi$ over $F$, if $\varphi_{K}$ is isotropic, then $\varphi$ is weakly isotropic over $F$.

Proof. (1) $\Rightarrow$ (2) Let $\varphi$ be a quadratic form over $F$ such that $\varphi_{K}$ is isotropic. Then, trivially, $\varphi$ is indefinite at any semiordering of $K$. Thus, if (1) holds, then $\varphi$ is also indefinite at any semiordering of $F$.

$(2) \Rightarrow(1)$ Let $S$ be a semiordering of $F$. By [10, Lemma (1.24)], $S$ extends to $K$ if and only if, for any quadratic form $\varphi$ over $F$ which is definite at $S$, the form $\varphi_{K}$ is anisotropic. This condition is provided by (2), since every weakly isotropic form over $F$ must be indefinite at $S$.

The lemma provides us with a couple of trivial but nevertheless important examples of totally positive field extensions. First, by Springer's Theorem, any finite extension of odd degree is totally positive. Second, if $K$ is the rational function field $F(X)$ or the field of power series $F((X))$, then $K / F$ is totally positive.

Proposition 3.2. Let $K=F(\sqrt{d})$ with $d \in F^{\times}$. Then $K / F$ is totally positive if and only if $d \in \sum F^{\times 2}$. 
Proof. If $d$ is not a sum of squares, then by the Artin-Schreier Theorem there exists an ordering $P$ of $F$ such that $d \notin P$. This ordering, which in particular is a semiordering, cannot extend to any semiordering on $K$, since $d$ is a square in $K$ and thus contained in every semiordering of $K$.

Assume now that $d \in \sum F^{\times 2}$. Let $\varphi$ be a quadratic form over $F$ such that $\varphi_{K}$ is isotropic. If $\varphi$ is anisotropic over $F$, then it follows that $\varphi$ contains a scalar multiple of $\langle 1,-d\rangle$, the norm form of $K / F$ (cf. [11]); as $d \in \sum F^{\times 2}$, the form $\varphi$ must be weakly isotropic over $F$ in this case. By the above lemma, this shows that $K / F$ is totally positive.

The smallest field extension of $F$ in which every totally positive element is a square is called the pythagorean closure of $F$; let us denote it by $\widetilde{F}$.

Corollary 3.3. $\widetilde{F} / F$ is a totally positive extension.

Proof. $\widetilde{F} / F$ is equal to the direct limit of all finite extensions $K / F$ where $K=$ $F_{m} \supset F_{m-1} \supset \cdots \supset F_{0}=F$ and where $F_{i}=F_{i-1}\left(\sqrt{d_{i}}\right)$ for some $d_{i} \in$ $\sum\left(F_{i-1}\right)^{\times 2}(i=1, \ldots, m)$; using the proposition and induction on $m$ we conclude that each such $K / F$ is a totally positive extension. Hence, the same holds for the direct limit of all these extensions.

Theorem 3.4. Let $\varphi$ be a quadratic form over $F$ with $\operatorname{dim}(\varphi) \geq 2$. Then $F(\varphi) / F$ is totally positive if and only if $\varphi$ is weakly isotropic over $F$.

Proof. Let $K=F(\varphi)$. Note that $\varphi_{K}$ is isotropic. Hence, if $K / F$ is totally positive, then $\varphi$ is weakly isotropic over $F$ by (3.1). To show the converse implication, let $\widetilde{F}$ denote the pythagorean closure of $F$. Assume that $\varphi$ is weakly isotropic over $F$. Then $\varphi_{\widetilde{F}}$ is isotropic and thus $\widetilde{F}(\varphi)$ is a rational function field (in $\operatorname{dim}(\varphi)-2$ variables) over $\widetilde{F}$. In particular $\widetilde{F}(\varphi) / \widetilde{F}$ is totally positive. Furthermore, $\widetilde{F} / F$ is totally positive by (3.3). Therefore, also the extension $\widetilde{F}(\varphi) / F$ is totally positive, and so must be $K / F$, which is contained in $\widetilde{F}(\varphi) / F$.

Remark 3.5. This result should be compared with a result on the extendibility of orderings due to Elman-Lam-Wadsworth. With the same notation as in the theorem, every ordering of $F$ extends to an ordering of $K=F(\varphi)$ if and only if $\varphi$ is totally indefinite, i.e. indefinite at every ordering of $F$. This is immediate from [3, Theorem 3.5.].

In the case of a quadratic extension $K / F$, the fact that all orderings of $F$ extend to orderings of $K$ is equivalent with the corresponding statement for semiorderings. This follows from (3.2). However, the two statements are not equivalent for arbitrary extensions.

Example 3.6. Let $F$ be a real field which is not a $S A P$-field. Let $\varphi$ be a totally indefinite form over $F$ which is not weakly isotropic. In fact, since $F$ is not a $S A P$-field, there exist $a, b \in F^{\times}$such that the form $\varphi=\langle 1, a, b,-a b\rangle$ is strongly anisotropic, while it is obviously totally indefinite. By [3, Theorem 3.5.], every ordering of $F$ extends to an ordering of $K=F(\varphi)$. However, (3.1) applied to $\varphi$ shows that $K / F$ is not totally positive.

Note that in this example $K / F$ is an extension of transcendence degree at least 2 . 
Question 3.7. Let $K / F$ be a field extension of transcendence degree $\leq 1$ (e.g. an algebraic extension) such that every ordering of $F$ extends to $K$. Is then $K / F$ a totally positive extension?

\section{The pythagorean index}

In this section, we will consider central simple algebras over a field. For the basic facts about these objects, I refer to [11, Chap. 8] and [8]. An important invariant of a central simple algebra is its (Schur-) index. Here I want to introduce a relative of this invariant, the pythagorean index, which will turn out to be useful for the study of weakly isotropic forms.

Let $A$ denote a central simple $F$-algebra. For a field extension $K / F$, let $A_{K}$ denote the central simple $K$-algebra $A \otimes_{F} K$. Recall that $\widetilde{F}$ is the pythagorean closure of $F$. Let $\operatorname{pind}(A)=\operatorname{ind}\left(A \otimes_{F} \widetilde{F}\right)$ be called the pythagorean index of $A$. For any field extension $K / F$ and any central simple $F$-algebra $A$, one has $\operatorname{pind}\left(A_{K}\right) \leq \operatorname{pind}(A)$, since $\widetilde{F} \subset \widetilde{K}$. What conditions on an extension $K / F$ are sufficient to have equality $\operatorname{pind}\left(A_{K}\right)=\operatorname{pind}(A)$ ?

Conjecture 4.1. Let $K / F$ be a totally positive extension. For any central simple $F$-algebra $A$ over $F$ of exponent 2 , one has $\operatorname{pind}\left(A_{F}\right)=\operatorname{pind}\left(A_{K}\right)$.

While I don't have an argument for this in the case of a finite extension of odd degree, I can confirm the conjecture in some other cases.

Proposition 4.2. Let $K / F$ be a field extension of one of the following types:

(1) $K=F(\sqrt{d})$, a quadratic extension where $d \in \sum F^{\times 2}$

(2) $K=F(X)$, the rational function field in one variable.

(3) $K=F((X))$, the field of power series in one variable.

Then for any central simple $F$-algebra $A$, one has $\operatorname{pind}\left(A_{F}\right)=\operatorname{pind}\left(A_{K}\right)$.

Proof. Case (1) is obvious, since $\widetilde{K}=\widetilde{F}$. In case (3), we have $\widetilde{K}=\widetilde{F}((X))$ and we know that there is no index reduction from $\widetilde{F}$ to $\widetilde{F}((X))$. This settles case (3), and then case (2) obviously follows.

Theorem 4.3. Let $\varphi$ be a weakly isotropic form over $F$ and $K=F(\varphi)$. Then for any central simple $F$-algebra $A$, one has pind $\left(A_{K}\right)=\operatorname{pind}(A)$.

Proof. We can assume that $\operatorname{dim}(\varphi) \geq 2$. If $\varphi$ is isotropic, then $K$ is a rational function field in $(\operatorname{dim}(\varphi)-2)$ variables over $F$ and the statement thus follows from case (2) of the last proposition. Assume now that $\varphi$ is anisotropic. Let $A$ be a central simple $F$-algebra. We have $\operatorname{pind}(A)=\operatorname{ind}\left(A_{\widetilde{F}}\right)=\operatorname{pind}\left(A_{\widetilde{F}}\right)$. Furthermore, since $\varphi$ is weakly isotropic, $\varphi_{\widetilde{F}}$ is isotropic. Applying the isotropic case, which we have already checked, to the extension $\widetilde{F}(\varphi) / \widetilde{F}$, we obtain that $\operatorname{pind}(A)=\operatorname{pind}\left(A_{\tilde{F}}\right)=\operatorname{pind}\left(A_{\widetilde{F}(\varphi)}\right)$. Since $K$ is contained in $\widetilde{F}(\varphi)$, we conclude that $\operatorname{pind}(A)=\operatorname{pind}\left(A_{K}\right)$. 


\section{The $\hat{u}$-invariant}

The invariant $\hat{u}(F)$ gives an isotropy criterion for weakly isotropic forms $\varphi$ over $F:$ if $\operatorname{dim}(\varphi)>\hat{u}(F)$, then $\varphi$ must be isotropic. By trivial arguments, we had the inequalities $u(F) \leq \hat{u}(F) \leq \widetilde{u}(F)$. In this section, I first give sufficient conditions to have equality between two of these invariants and then determine the range of possible even values for $\hat{u}(F)$ when $u(F)$ is given.

Recall that $F$ is a $S A P$-field if and only if every totally indefinite form over $F$ is weakly isotropic (cf. [10]). In this case we obtain from the definitions that $\hat{u}(F)=\widetilde{u}(F)$.

Proposition 5.1. If $\widetilde{u}(F)<\infty$, then $\hat{u}(F)=\widetilde{u}(F)$.

Proof. If $\tilde{u}(F)<\infty$, then it is easy to see that $F$ is a $S A P$-field. (In fact, $F$ then is even a so-called $E D$-field.) Therefore, $\hat{u}(F)=\widetilde{u}(F)$ in this case.

Thus, the $\hat{u}$-invariant may only provide new information for real fields $F$ which are not $S A P$-fields and for which, in particular, $\widetilde{u}(F)$ is infinite. Admittedly, it seems that in all natural examples of real fields $F$ other than $S A P$-fields, one will have $\hat{u}(F)=\tilde{u}(F)=\infty$. Examples of fields $F$ with $\hat{u}(F)<\tilde{u}(F)=\infty$ will be constructed below.

Example 5.2. Let $k$ be a real field which is not pythagorean, hence with an element $d \in \sum k^{\times 2} \backslash k^{\times 2}$. Let $F=k(t)$. For any $m \in \mathbb{N}$, the form $m \times\langle 1\rangle \perp\langle t,-d t\rangle$ over $F$ is anisotropic, but weakly isotropic. This shows that $\hat{u}(F)=\widetilde{u}(F)=\infty$. Obviously, the same argument applies to $F=k((t))$. Here, if one wants in addition $k((t))$ to be a $S A P$-field, then it is only necessary to choose $k$ uniquely ordered. On the other hand, $k(X)$ cannot be a $S A P$-field if the element $d \in \sum k^{\times 2} \backslash k^{\times 2}$ exists, since $\left\langle 1, X, X^{2}-d,-X\left(X^{2}-d\right)\right\rangle$ then is a totally indefinite, strongly anisotropic form over $k(X)$.

Proposition 5.3. If $u(F) \leq 2$, then $\hat{u}(F)=u(F)$.

Proof. If $u(F)=0$ then $F$ is real pythagorean, hence every anisotropic form over $F$ is already strongly anisotropic, which implies that $\hat{u}(F)=0$ as well. If $u(F)=1$, then $F$ is quadratically closed, hence $\hat{u}(F)=u(F)=1$.

Assume now that $u(F)=2$. Since $u(F)=2$, every totally positive element in $F$ can be written as a sum of two squares in $F$. Given an arbitrary anisotropic, weakly isotropic form $\varphi$ over $F$, it follows that already $2 \times \varphi$ is isotropic. Therefore any such form $\varphi$ with $\operatorname{dim}(\varphi) \geq 2$ contains a 2 -dimensional torsion form $\beta$ (cf. [2]). Since $u(F)=2$, this form $\beta$ is universal and thus cannot be strictly contained in any anisotropic form, so that we may conclude that $\operatorname{dim}(\varphi)=2$. This shows that $\hat{u}(F)=2$.

Given a quadratic form $\varphi$ over $F$, let $C(\varphi)$ denote its Clifford algebra (cf. [6, Chap. V]).

Theorem 5.4. For any $m, n \in \mathbb{N}$ with $2 \leq m \leq n$, there is a real field $K$ such that $l^{3} K$ is torsion-free, $u(K)=2 m, \hat{u}(K)=2 n$, and $\tilde{u}(K)=\infty$. 
Proof. We start with a real field $k$ such that there exist central simple $k$-algebras $A$ and $B$, and quadratic forms $\psi_{A}, \psi_{B}$, and $\rho$, subject to the following requirements:

- $\rho$ is totally indefinite and strongly anisotropic over $k$;

- $\psi_{A}$ is weakly isotropic, $\operatorname{dim}\left(\psi_{A}\right)=2 n$, and $\psi_{A} \in I^{2} k$;

- $C\left(\psi_{A}\right) \cong M_{2}(A), \operatorname{ind}(A)=2^{n-1}$, and $\operatorname{pind}(A)=2^{n-2}$;

- $\psi_{B}$ is torsion, $\operatorname{dim}\left(\psi_{B}\right)=2 m$, and $\psi_{B} \in I^{2} k$

- $C\left(\psi_{B}\right) \cong M_{2}(B)$ and $i n d(B)=2^{m-1}$.

Note that, as a consequence of these conditions, $A$ and $B$ are division algebras, and $\psi_{A}$ and $\psi_{B}$ are anisotropic. Moreover, since $M_{2}(B) \cong C\left(\psi_{B}\right)$ and since $\psi_{B}$ is torsion, it follows that $\operatorname{pind}(B)=1$.

We now choose a field extension $K / k$ such that all the conditions on the Schur indices and the pythagorean indices of $A$ and $B$ are still satisfied after scalar extension to $K$ and such that $\rho_{K}$ is still strongly anisotropic, and such that, on the other hand, for any anisotropic form $\varphi$ over $K$ with $\operatorname{dim}(\varphi) \geq 2$, at least one of these conditions is violated for the field $K(\varphi)$. It follows from $[1,(6.1)]$ that such a field exists. Such an extension can, in fact, easily be constructed using the Merkurjev method (cf. [4]).

Since $\rho_{K}$ is strongly anisotropic and totally indefinite over $K$, we know that $K$ cannot be a $S A P$-field, so in particular $\tilde{u}(K)=\infty$.

Let $\varphi$ be an anisotropic quadratic form over $K$. If $\varphi$ is weakly isotropic over $K$, then the pythagorean indices of $A$ and $B$ are still conserved after scalar extension to $K(\varphi)$, by (4.3). Moreover, $K(\varphi) / K$ is a totally positive extension, whence $\rho_{K(\varphi)}$ is still strongly anisotropic and, certainly, totally indefinite over $K(\varphi)$. Therefore, there is an index reduction for at least one of the algebras $A_{K}$ and $B_{K}$ when they are extended from $K$ to $K(\varphi)$. By Merkurjev's Criterion (cf. [12, Théorème $1]$ ), this implies that $\operatorname{dim}(\varphi) \leq 2 n$. So far, this argument shows that $\hat{u}(K) \leq 2 n$. On the other hand, $\operatorname{dim}\left(\left(\psi_{A}\right)_{K}\right)=2 n$ and $\left(\psi_{A}\right)_{K}$ is still anisotropic over $K$ as $\operatorname{ind}\left(A_{K}\right)=2^{n-1}$, but also weakly isotropic. Therefore, we have $\hat{u}(K)=2 n$.

Assume now that $\varphi$ is even a torsion form over $K$. Let $r \in \mathbb{N}$ be such that $\operatorname{dim}(\varphi)=2 r$. Over the pythagorean closure $\widetilde{K}$ we get $\varphi_{\widetilde{K}} \cong r \times \mathbb{H}$, whence $C\left(\varphi_{\widetilde{K}}\right) \cong M_{2^{r}}(\widetilde{K})$.

Suppose that ind $\left(A_{K(\varphi)}\right)<2^{n-1}=\operatorname{ind}\left(A_{K}\right)$. Then Merkurjev's Criterion implies that $M_{2}\left(A_{K}\right) \cong C\left(\varphi_{K}\right) \otimes_{K} D$ for a central simple $K$-algebra $D$. Since $\operatorname{pind}\left(A_{K}\right)=2^{n-2}$, we conclude that $M_{2}\left(A_{\widetilde{K}}\right)$ contains no matrix algebra $M_{s}(\widetilde{K})$ where $s>4$. As $M_{2^{r}}(\widetilde{K}) \cong C\left(\varphi_{\widetilde{K}}\right)$ is contained in $M_{2}\left(A_{\widetilde{K}}\right)$, it follows that $r \leq 2$, whence $\operatorname{dim}(\varphi) \leq 4$. This shows that $i n d\left(A_{K(\varphi)}\right)=i n d\left(A_{K}\right)$ for any torsion form $\varphi$ with $\operatorname{dim}(\varphi)>4$

Furthermore, if ind $\left(B_{K(\varphi)}\right)<2^{m-1}=$ ind $\left(B_{K}\right)$, Merkurjev's Criterion implies that $\operatorname{dim}(\varphi) \leq 2 m$. Note that $4 \leq 2 m$.

These arguments together with the choice of $K$ imply that we have $\operatorname{dim}(\varphi) \leq$ $2 m$ for any anisotropic torsion form $\varphi$ over $K$, proving that $u(K) \leq 2 m$. But $\operatorname{dim}\left(\left(\psi_{B}\right)_{K}\right)=2 m$ and $\left(\psi_{B}\right)_{K}$ is torsion and anisotropic, since $\operatorname{ind}\left(C\left(\left(\psi_{B}\right)_{K}\right)\right)=$ $2^{m-1}$. Therefore we have $u(K)=2 m$.

Furthermore, if $\varphi \in I^{3} K$ and if $\varphi$ is weakly isotropic, then no condition would be violated over $K(\varphi)$. This shows that $I^{3} K$ contains no anisotropic weakly isotropic form. In particular, $I^{3} K$ is torsion-free. 
Remarks 5.5. (1) If $\tilde{u}(F)<\infty$, then $u(F) \leq \hat{u}(F)=\widetilde{u}(F)$ by (5.1). In [5] the complete list of possible pairs $(u(F), \widetilde{u}(F))$ for real fields $F$ with $I^{3} F$ torsionfree has been determined.

(2) Let $F$ be a real field. Then any torsion form over $F$ is of even dimension. Hence $u(F)$ is either an even number or infinite. It may be asked whether the same is true for $\hat{u}(F)$, at least under the additional assumption that $I^{3} F$ is torsion-free.

Acknowledgements. This research was supported by the European RTN Network 'Algebraic K-Theory, Linear Algebraic Groups, and Related Structures' (HPRN-CT-200000287) and by the Swiss National Science Foundation (Grant No. 200020-100229/1). I am grateful to the referee for several valuable remarks on a preliminary version of this paper.

\section{References}

[1] Becher, K.J.: Supreme Pfister forms. Comm. Alg. 32, 217-241 (2004)

[2] Elman, R., Lam, T.Y.: Quadratic forms and the $u$-invariant I. Math. Z. 131, 283-304 (1973)

[3] Elman, R., Lam, T.Y., Wadsworth, A.R.: Orderings under field extensions. J. Reine Angew. Math. 306, 7-27 (1979)

[4] Hoffmann, D.W.: Isotropy of quadratic forms and field invariants. Cont. Math. 272, 73-101 (2000)

[5] Hoffmann, D.W.: Dimensions of Anisotropic Indefinite Quadratic Forms, I. Documenta Math., Quadratic Forms LSU 2001 183-200 (2001)

[6] Lam, T.Y.: Introduction to quadratic forms over fields. Graduate Studies in Mathematics, 67. American Mathematical Society, Providence, RI, 2005.

[7] Pfister, A.: Quadratic Forms with Applications to Algebraic Geometry and Topology. London Math. Soc. Lect. Notes 217. Cambridge University Press, 1995

[8] Pierce, R.S.: Associative algebras. Graduate Texts in Mathematics 88, SpringerVerlag, New York, 1982

[9] Prestel, A.: Remarks on the Pythagoras and Hasse number of real fields. J. Reine Angew. Math. 303/304, 284-294 (1978)

[10] Prestel, A.: Lectures on Formally Real Fields. Lecture Notes in Math. 1093, SpringerVerlag, Berlin, 1984

[11] Scharlau, W.: Quadratic and Hermitian forms. Grundlehren Math. Wiss. 270, Springer-Verlag, Berlin, 1985

[12] Tignol, J.-P.: Réduction de l'indice d'une algèbre simple centrale sur le corps des fonctions d'une quadrique. Bull. Soc. Math. Belgique Sér. A 42, 735-745 (1990) 